\title{
Interaction of Laser Light and Electrons with Nanotubes
}

\author{
H. R. Sadeghpour* and Brian E. Granger** \\ ${ }^{1}$ ITAMP, Harvard-Smithsonian Center for Astrophysics, Cambridge, Massachusetts 02138, USA
}

Received August 27, 2003; accepted November 24, 2003

PACS Ref: $34.60 .+\mathrm{z}, 36.10 .-\mathrm{k}, 34.80 . \mathrm{Lx}$

\begin{abstract}
Electronic states in designed geometries are attracting considerable attention. In this work, it is shown that it may be possible to induce ultrafast mechanical rotation in nanotubes using circularly polarized light. We predict a novel set of Rydberg-like electronic states around nanotubes having long lifetimes and exotic properties. Preliminary work on the interaction of slow electrons with an array of parallel nanotubes is discussed.
\end{abstract}

\section{Introduction}

Technological leaps of the last two decades have opened windows of opportunity for designing and controlling electronic states in novel systems. The fabrication of quantum dots in semiconductor devices has led to the invention of single-electron transistors $[1,2]$ and controllable single photon emitters [3]. Multielectron shell structure has emerged from studies of magnetic-field induced transitions in quantum dots [4]. Such designer atoms are ideal settings for the control and manipulation of electronic states.

Carbon nanotubes were first synthesized in 1991 by Iijima [5] as graphitic carbon needles, ranging from 4 to 30 nanometers in diameter and up to one micron in length using carbon arc evaporation and condensation in a soot. Carbon nanotubes have remarkable electrical and mechanical properties. Depending on their microscopic structure, they can be made to conduct electricity and heat, inhibit the conduction of electricity, or act as semiconductors. Experiments have found that carbon nanotubes have an average modulus of elasticity of about 1000 gigapascals (GPa) [6] (note that the Young's modulus for typical steel is a few hundred GPa). Thus, carbon nanotubes are not only more flexible than steel, they are a few times stronger as well. Nanotubes can be used effectively act as chimneys, conducting heat from integrated circuits and hence cooling them. As the largest non-biological molecules, carbon nanotubes inherit their remarkable mechanical properties from the strong carbon covalent bonds, where two electrons are shared with the nearest neighbor carbon atoms. Furthermore, because carbon nanotubes are well rounded, they do not have discontinuities and/or dislocations to initiate fractures and form cracks.

A carbon nanotube (CNT) is constructed by rolling a graphene sheet, defined by its primitive lattice vectors, $\boldsymbol{a}_{1}$ and $\boldsymbol{a}_{2}$, along a vector $\boldsymbol{C}_{h}$, the so-called chiral vector, around an axial or translation vector $\boldsymbol{T}$ (see Fig. 1).

\footnotetext{
* e-mail: hsadeghpour@cfa.harvard.edu

** e-mail: bgranger@scu.edu Permanent address: Dept. of Physics, Santa Clara University Santa Clara, CA 95053, USA
}

The chiral vector,

$\boldsymbol{C}_{h}=n \boldsymbol{a}_{1}+m \boldsymbol{a}_{2} \equiv(n, m)$,

and translation vector,

$\boldsymbol{T}=\left[\frac{(2 m+n)}{d_{R}} \boldsymbol{a}_{1}-\frac{(2 n+m)}{d_{R}} \boldsymbol{a}_{2}\right]$

are determined by two lattice indices, $n$ and $m$. Here,

$d_{R}=\left(\begin{array}{ll}3 d_{n m} & \text { if } n-m=3 q d_{n m} \\ d_{n m} & \text { if } n-m \neq 3 q d_{n m}\end{array}\right)$

where $q$ is an integer, $d_{R}$ is the greatest common divisor of $2 n+m$ and $2 m+n$, and $d_{n m}$ is the greatest common divisor of $n$ and $m$.

The diameter of an $(n, m)$ CNT is $d=C_{h} / \pi$, where $C_{h}=$ $\sqrt{3} a_{c-c}\left[m^{2}+n^{2}-m n\right]^{1 / 2}$ is the magnitude of the chiral vector, and $a_{c-c}=1.42$ is the carbon-carbon bond length. The chirality of a nanotube is characterized by the chiral angle,

$\theta=\tan ^{-1}[\sqrt{3} m /(m+2 n)]$,

which is the angle between the vectors $\boldsymbol{C}_{h}$ and $\boldsymbol{a}_{1}$.

Nanotubes having either (but not both) $m=0$ or $n=0$ have $\theta=0^{\circ}$ and are called zigzag nanotubes due to the zigzag nature of the bonds at the tube ends. Likewise, tubes with $n=m$ have $\theta=30^{\circ}$ and are called armchair tubes [7,8]. All other tubes with $n \neq m$ are called chiral nanotubes. One of the most remarkable properties of carbon nanotubes is that depending on their chirality, conducting, semiconducting or insulating nanotubes are possible [9]. For armchair and zigzag nanotubes, and when $(n-m)$ is divisible by 3 , the tube conducts. Otherwise semiconducting nanotubes form. For chiral nanotubes, i.e. $(m \neq n)$, if $(2 n+m) / 3$ or equivalently $(n-m) / 3$ is an integer, metallic tubes form. Experiments [10,11] have observed that the IV curve (current vs. voltage) for a $(11,2)$ CNT is a monotonically increasing function of the bias voltage, indicating a metallic structure; a fact that is also consistent with the $(2 n+m) / 3=8$ being an integer, see Fig. 2. The measured angle was $\theta=-8.0 \pm 0.5^{\circ}$, whereas the calculated value is $\theta=-8.2^{\circ}$.

The number of hexagons, $N$, per unit tube cell can be written down as

$N=2\left(n^{2}+m^{2}+n m\right) / d_{R}$

with $d_{R}$ defined previously. For instance, the single-walled $(10,10)$ carbon nanotube has $N=20$ and since each 
(a)
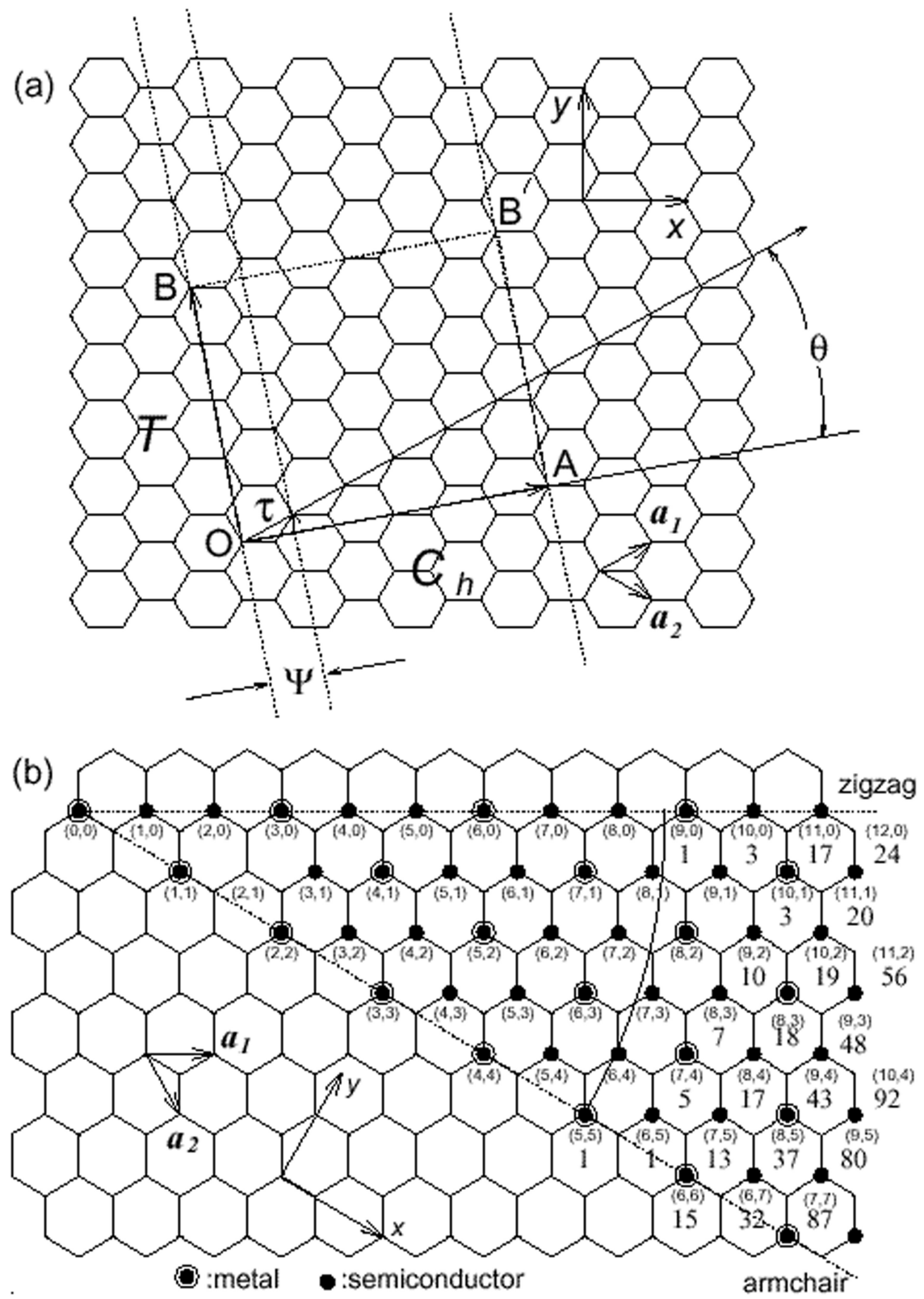

Fig. 1. A graphene honeycomb lattice showing the primitive vectors and the chiral and translation vectors. From Ref. [7].

hexagon has two full carbon atoms, the total number of atoms in one unit of a $(10,10) \mathrm{CNT}$ is $N_{c}=40$. For a $(9,0)$ CNT, $N=18$. There are 10 valence and conduction electrons, with two non-degenerate and eight doublydegenerate levels; for a total of 18 states (see Fig. 3). Both the $(10,10)$ armchair and $(9,0)$ zigzag nanotubes are conducting nanotubes.

The one-dimensional energy dispersion relations in Fig. 3 are labeled by their irreducible representations of the point groups, $D_{n d}$ or $D_{n h}$, depending upon whether there are even or odd numbers of bands at the $\Gamma$ point at $k=0$ respectively. The $a$-bands are nondegenerate and the $e$ bands are doubly-degenerate. The $X$ points for armchair nanotubes refer to $k= \pm \pi / \sqrt{3} a_{c-c}$ and for zigzag tubes to $k= \pm \pi / 3 a_{c-c}$. These dispersion curves clearly show the crossing of the valence and conductions bands at the Fermi energy, $E_{F}$, for the $(5,5)$ and $(9,0)$ CNTs and the gap between the bands at $E_{F}$ for the $(10,0)$ CNT. The $(5,5)$ and $(9,0)$ CNTs are considered zero-gap semiconductors that exhibit metallic conduction at finite temperatures. It has been shown that the density of electronic states near the Fermi energy is finite for all metallic nanotubes, independent of their diameter or chirality, and is zero for semiconducting nanotubes. The density of states as a function of energy also shows singular peaks at the valence and conduction band edges, the so-called van Hove singularities, reminiscent of $1 \mathrm{D}$ behavior in semiconductors [13]. Because of these singularities in the density of states, optical absorption is expected at frequencies resonant with the energy separations of the van Hove peaks. Indeed, a recent optical spectroscopy of more than 30 different semiconducting nanotubes [12] promises to become an exacting probe of composition of bulk nanotubes.

\section{Photoabsorption in carbon nanotubes: Mechanical rotation}

The vibrational modes of carbon nanotubes have been investigated by several workers and the phonon dispersion energy curves have been calculated for a large number of 


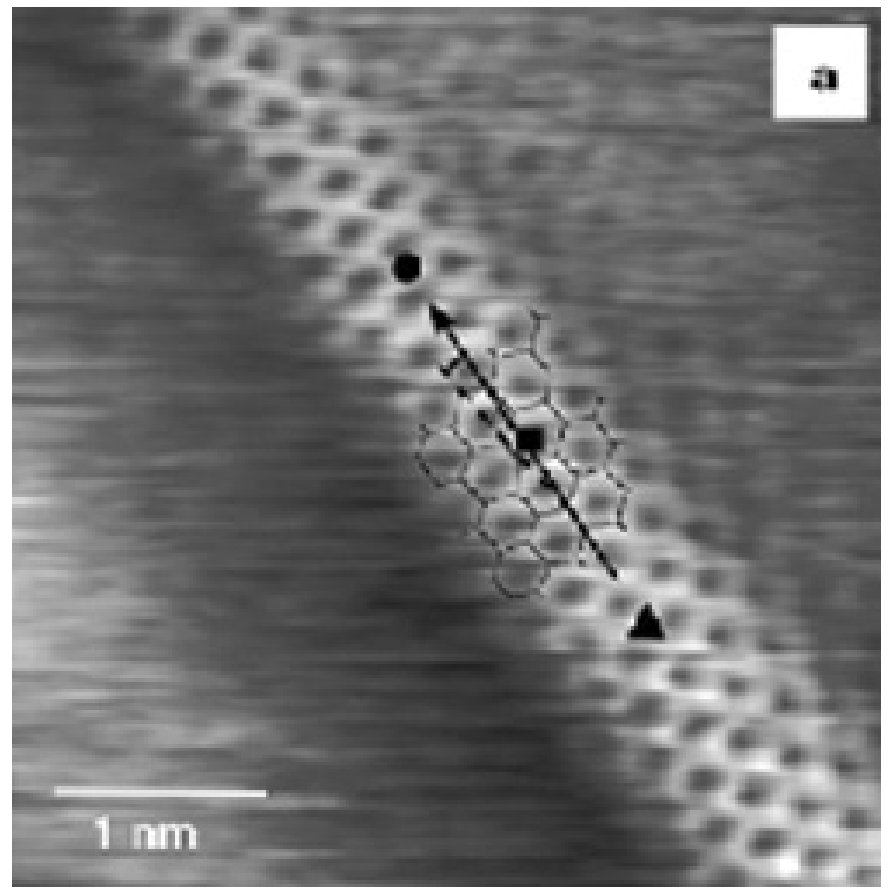

Fig. 2. The honeycomb nature of single-walled CNT. From an STM measurements of Odom et al. [10].

CNTs using the zone-folding method $[8,13]$. Of particular interest are two optically-active infrared (IR) phonon modes at $\omega_{A_{2 u}}=870 \mathrm{~cm}^{-1}$ and $\omega_{E_{1 u}}=1580 \mathrm{~cm}^{-1}$. Both of the IR-active modes absorb photons and carry angular momentum. Figure 4, gives the dipolar pattern of the carbon atom displacements [14] in the doubly-degenerate $A_{2 u}$ phonon mode for a $(10,10) \mathrm{CNT}$.

We have investigated the possibility of inducing ultrafast asynchronous rotation in nanotubes by exciting this mode with circularly polarized light [15] propagating along the tube. We found that with realistic oscillator strength and density of optical phonon modes, the IR-active phonons can absorb angular momentum at a rate of $\dot{L}_{A_{2 u}}=$ $\hbar \dot{n}_{A_{2 u}}^{+} \approx 2.5 \times 10^{-29} \mathrm{Nm}$. The observed decay lifetime of the $A_{2 u}$ mode is roughly $2.2 \mathrm{ps}$, giving a steady-state angular momentum of $L_{A_{2 u}}=\hbar \Delta n_{A_{2 u}}^{+}=\dot{L}_{A_{2 u}} \tau_{A_{2 u}} \approx$ $5.2 \times 10^{-41} \mathrm{Js}$. This angular momentum is transferred to the tube following the two-acoustical phonon decay of the $A_{2 u}$ mode near the $k=0$ branch.

$(5,5)$

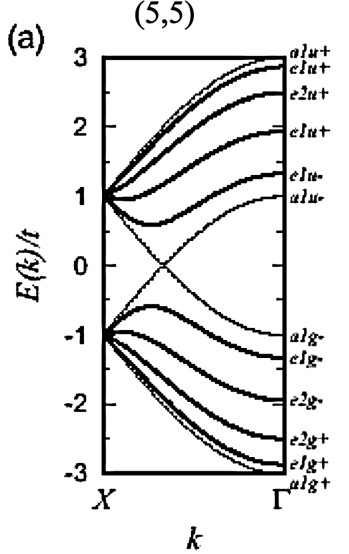

$(9,0)$

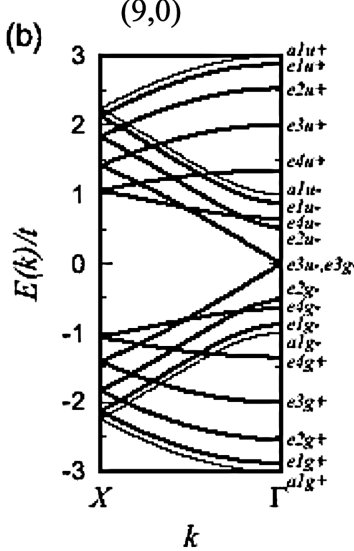

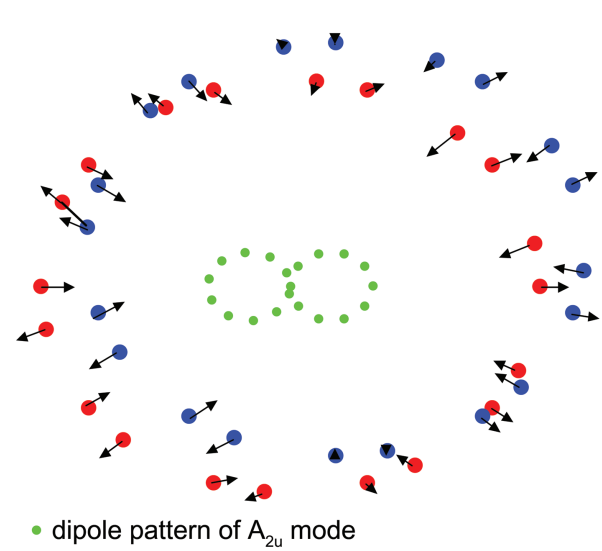

Fig. 4. Ir-active $A_{2 u}$ phonon mode in $(10,10)$ carbon nanotube at $\omega_{A_{2 u}}=870 \mathrm{~cm}^{-1}$.

Upon calculating the tube's principal moments of inertia [16], the nanotubes are found to rotates with a frequency of $\omega_{\text {rot }} \approx 28 \mathrm{GHz}$. The centrifugal acceleration on the tube surface is thus huge: $a=r \omega_{\text {rot }}^{2}=0.5 \times 10^{12} \mathrm{~m} / \mathrm{s}^{2} \approx 10^{11} \mathrm{~g}$. This value is $2-5$ orders of magnitude larger than the fastest centrifuges, but because the resulting force on each $\mathrm{C}$ atom $(F \approx 13 \mu \mathrm{eV} / \AA)$ is negligible compared with typical molecular bond force of $1 \mathrm{eV} / \AA$, the distortion of the tube due to rotation is minimal. Because CNTs, even semiconducting ones, are far more polarizable along their axis, than in the transverse direction [17], we proposed that an asymmetric light-force trap could suspend a nanotube [18] in vacuum, making it possible to spin it at these high frequencies. We also investigated the mechanical stability of the nanotube rotation by calculating the different resonant whirl and flexural modes for rigid and non-rigid rotations of the nanotube; it is encouraging that these modes have excitation frequencies far below $\omega_{\text {rot }}$. This proof-of-concept study shows that nanotubes with their particular mechanical and electrical properties could form parts of nanoscopic motors, centrifuges or stabilizers.

\section{Rydberg-like electronic states near nanotubes}

Rydberg states are ubiquitous in atomic and molecular physics [19]. Extreme Rydberg states of hydrogen and heavier elements, $\mathrm{Mg}$ and $\mathrm{Si}$ for example, have been detected in the solar atmosphere and in the interstellar

Fig. 3. The calculated electronic band structure of several carbon nanotubes [8].

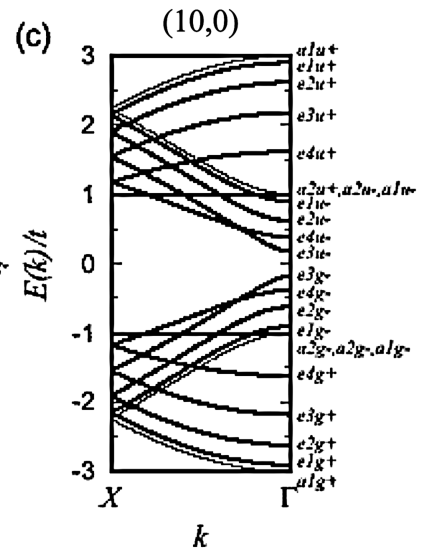


medium [20,21]. The recombination of electrons and ions, through radiative recombination or three-body collisions, into highly-excited Rydberg states is an important neutralization process in laboratory and astrophysical plasmas [22]. The creation of cold antihydrogen atoms in nested Penning traps [23] most likely proceeds through the recombination of antiprotons and positrons into Rydberg states. Advances in cooling techniques have led to creation of ultracold neutral plasmas through the photoionization of laser-cooled atoms [24]. Rydberg xenon atoms with principal quantum numbers as high as $n=60$ and lifetimes of hundreds of microseconds have been formed in the expansion of a plasma [24]. Collisions between ultracold neutral and Rydberg atoms in magneto-optical traps have been predicted to form exotic classes of Rydberg molecules dubbed "trilobite" and "butterfly" molecules [25]. Lastly, Rydberg states of $\mathrm{C}_{60}$ have recently been observed [26].

Rydberg electronic states have also been observed above conducting surfaces. This is made possible by the attractive interaction of an electron with its image charge. The resulting interaction potential for an electron at a distance $z$ above a flat surface having a dielectric constant $\varepsilon$, is: $V(z)=-e^{2} / 4 z(\varepsilon-1 / \varepsilon+1)$ [31]. Because of the Coulomb interaction, this potential supports an infinite number of "image states" that form the familiar Rydberg-series, $E_{n}=-13.60 / 16 n^{2}(\varepsilon-1 / \varepsilon+1)^{2} \mathrm{eV}$, where $n$ is the principal quantum number.

Femtosecond two-photon pump-probe spectroscopy of electronic image states near $\mathrm{Cu}(100)$ surfaces has revealed quantum beats due to the interference of different eigencomponents in the resulting electronic wavepackets above the surface [27]. The binding energies of these states are roughly $15-40 \mathrm{meV}$ and their lifetimes are a few picoseconds. More typical lifetimes are tens of femtoseconds because above flat surfaces there is no angular momentum to keep the electron away from the surface. The collapse of these states into the surface is the primary reason for their short lifetimes. Similar experiments performed above molecular wires laid on surfaces [28], nano-particles [26,29], and one- and two-dimensional liquid $\mathrm{He}$ [30] demonstrate that extended image states are common in nanoscopic systems. All of these experiments on Rydberg states lead us to the question: are there Rydberg-like electronic excitations of conducting carbon nanotubes?

We have shown that conducting CNTs do indeed have Rydberg-like excitations. These Rydberg states form through the same physical mechanism as the Rydberg states above flat conducting surfaces; that is, through the electron's attraction to its image charge [32]. However, because an electron can have angular momentum about the nanotube axis, a centrifugal barrier can form. The result is that the electronic wavefunction is kept away from the surface of the tube, dramatically increasing the lifetimes of the states compared to their counterparts above flat surfaces, see Fig. 5.

We begin by treating a perfectly conducting nanocylinder, where the induced scalar potential due to an external charge $q$ is found by requiring the total scalar potential to

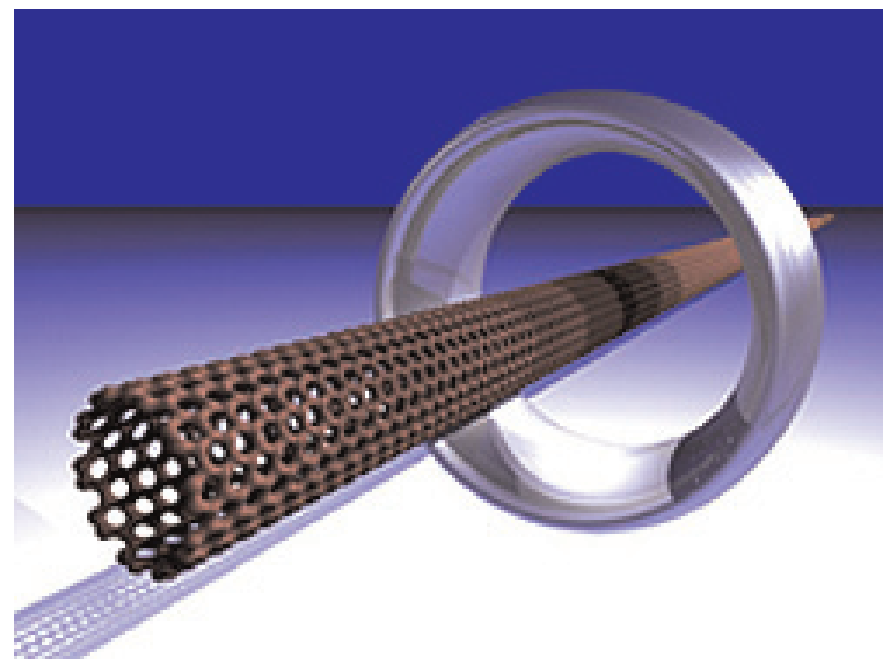

Fig. 5. A visualization is shown of an electron in a tubular image state around a $(10,10)$ metallic carbon nanotube. From Ref. [32].

vanish on the tube surface $(\rho=a)$ :

$$
\begin{aligned}
\Phi_{\text {ind }}(\rho, \varphi, z)= & -\frac{2 q}{\pi} \sum_{m=-\infty}^{m=+\infty} \int_{0}^{\infty} \mathrm{d} k \cos (k z) \exp (\mathrm{i} m \phi) \\
& \times \frac{I_{m}(k a)}{K_{m}(k a)} K_{m}\left(k \rho_{0}\right) K_{m}(k \rho) .
\end{aligned}
$$

We take the charge (the electron) to be located outside the tube at the position $\left(\rho_{0}, 0,0\right)$ and $\left(I_{m}(x), K_{m}(x)\right)$ are the regular and irregular modified Bessel functions. The electrostatic force between the electron and the conducting cylinder is calculated by differentiating Eq. (2) with respect to $\rho$ :

$$
\begin{aligned}
F\left(\rho_{0}\right) & =-q \partial \Phi_{\text {ind }} /\left.\partial \rho\right|_{\left(\rho_{0}, 0,0\right)} \\
& =\frac{2 q^{2}}{\pi a^{2}} \int_{0}^{\infty} \mathrm{d} x\left[A_{0}(x)+2 \sum_{m=1}^{\infty} A_{m}(x)\right] \\
A_{m}(x) & =\frac{I_{m}(x)}{K_{m}(x)} K_{m}\left(x \rho_{0} / a\right) x K_{m}^{\prime}\left(x \rho_{0} / a\right) .
\end{aligned}
$$

The potential energy $V\left(\rho_{0}\right)=-\int^{\rho_{0}} F(\rho) \mathrm{d} \rho$ can be calculated numerically from these expressions. Alternatively, physical intuition can be gained by an asymptotic analysis of the potential. The result,

$$
V\left(\rho_{0}\right) \sim \frac{q^{2}}{a} \operatorname{li}\left(\frac{a}{\rho_{0}}\right) \approx-\frac{q^{2}}{a} \frac{1}{\left(\rho_{0} / a\right) \ln \left(\rho_{0} / a\right)},
$$

is dominated by the $m=0$ term in Eq. (3) and is given in terms of the logarithmic integral $\operatorname{li}(x) \equiv \int_{0}^{x} \mathrm{~d} t / \ln (t)$. This long range behavior of the potential agrees with the expectation that at large distances the result for the conducting cylinder should lie somewhere between that of a conducting plane $(V \sim-1 / z)$ and a conducting sphere ( $V \sim-1 / r^{2}$, where $r$ is the radial distance).

The above expressions are valid for the case of a perfect cylindrical conductor, but can be extended to a nanocylinder with a finite anisotropic dielectric tensor $\varepsilon_{i j}$. Most carbon nanotubes have much smaller values of dielectric constants in the transverse direction, that is $\varepsilon_{x x}=\varepsilon_{y y} \ll \varepsilon_{z z}$. We have extended the above derivation to include an anisotropic dielectric function and have 


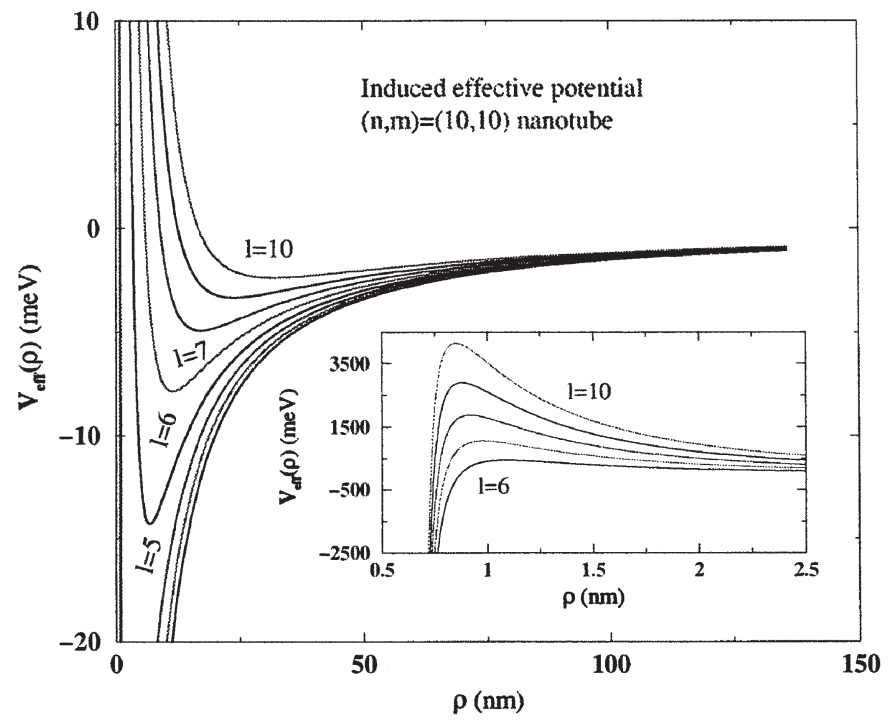

Fig. 6. The effective potential $V_{\text {eff }}(\rho)$ between an electron and a conducting nanotube as a function of the angular momentum, $l$. From Ref. [32].

found that as long as the dielectric function is large along the tube, i.e. $\varepsilon_{z z} \varepsilon_{x x} \gg 1$, the interaction potential is practically identical to the perfectly conducting case.

The total effective potential that an electron would "see" in front of a conducting nanotube is

$V_{\mathrm{eff}}(\rho)=V(\rho)+\frac{\left(l^{2}-1 / 4\right)}{2 m \rho^{2}}$

where $m$ is the mass of the electron. This potential for different values of the angular momentum, is shown in Fig. 6 for a $(10,10)$ carbon nanotube of radius $a=0.68 \mathrm{~nm}$. For moderate angular momenta $(l \gtrsim 6)$, the effective potential possesses extremely long-range wells that support bound states. The inset of Fig. 6 shows that high $(1-2 \mathrm{eV})$ potential barriers separate these wells from the tube surface. Indeed, for angular momentum values of $l<6$, no long-range wells exist so that low angular momentum states will exist close to the surface and have short lifetimes. However, because the radial overlaps between the high angular momentum and lower angular momentum states are small (they are localized at different radial distances) the decay of the high angular momentum states to ones with lower angular momentum through spontaneous radiative decay will be greatly suppressed.

The critical angular momentum, above which it is expected that long-range potential wells form is given in Fig. 7. The solid line identifies the demarcation between the two cases and some of the more common CNTs are also indicated in the figure. If the total wave function is written as $\Psi_{n, l, k}(\rho, \varphi, z)=\psi_{n, l}(\rho) \mathrm{e}^{\mathrm{i} / \varphi} \phi_{k}(z) / \sqrt{2 \pi \rho}$, the motion along the tube separates out so that the total energy becomes $E_{n, l, k}=E_{n, l}+E_{k}$. The longitudinal energy $E_{k}$ is either continuous $\left(E_{k}=k^{2} / 2\right)$ or quantized depending on whether the electron is localized in the $\hat{z}$ direction or not-a possible scenario would be to create metallicsemiconducting junctions by connecting different chirality nanotubes end-to-end. It is worth mentioning that the transverse energies, $E_{n, l}$, are no longer degenerate in $l$, as in the case of hydrogen atoms, and scale as $E_{n, l} \sim l^{-3}$ for fixed $n$. Figure 8 shows the $n=1$ transverse wavefunctions $\psi_{n=1, l}(\rho)$ for the potentials of Fig. 6. It is clear that even for

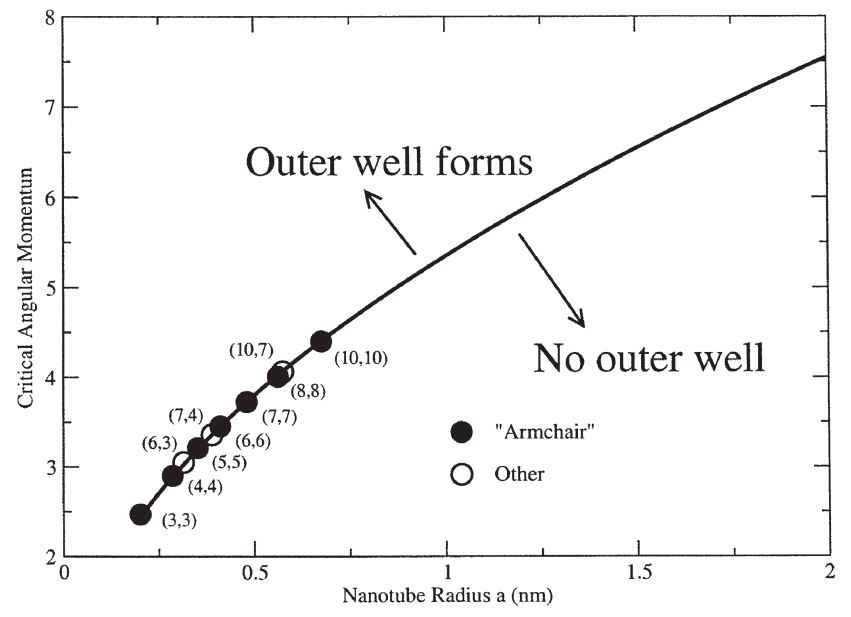

Fig. 7. The critical angular momentum needed to form the long-range wells.

moderate value of angular momentum, the electron is kept substantially away from the nanotube $(10-50 \mathrm{~nm})$ surface, with the distance of maximum probability scaling as $l^{3}$.

The decay of these states through the collapse of the wave function into the surface is highly suppressed, due to the existence of large repulsive centrifugal barriers. The lifetimes due to tunneling increase exponentially with $l$ and are substantially longer than those of image states above planar surfaces. Interaction with holes in the nanotube and the coupling of these states to the phonon modes in the tube can reduce their lifetimes. These decay mechanisms can be suppressed by operating at temperatures $(T<10 \mathrm{~K})$ lower than the transition energy between different image states. Spontaneous radiative transitions between different states also limit their lifetimes. The calculated lifetimes between $l_{f}=l_{i} \pm 1$ are found to be $5-10 \mathrm{~ms}$ and stimulated transitions due to blackbody radiation cut these lifetimes to probably hundreds of microseconds. Nevetheless, these lifetimes are at least several orders of magnitude longer than the image state lifetimes that have been observed to date.

Two likely mechanisms for forming these states are inverse photoemission (radiative recombination) and charge exchange with ultracold Rydberg atoms. The former process favors low-energy recombination with rate coefficients of $\alpha^{r r} \sim 10^{-13}-10^{-12} \mathrm{~cm}^{3} \mathrm{~s}^{-1}$. In the latter

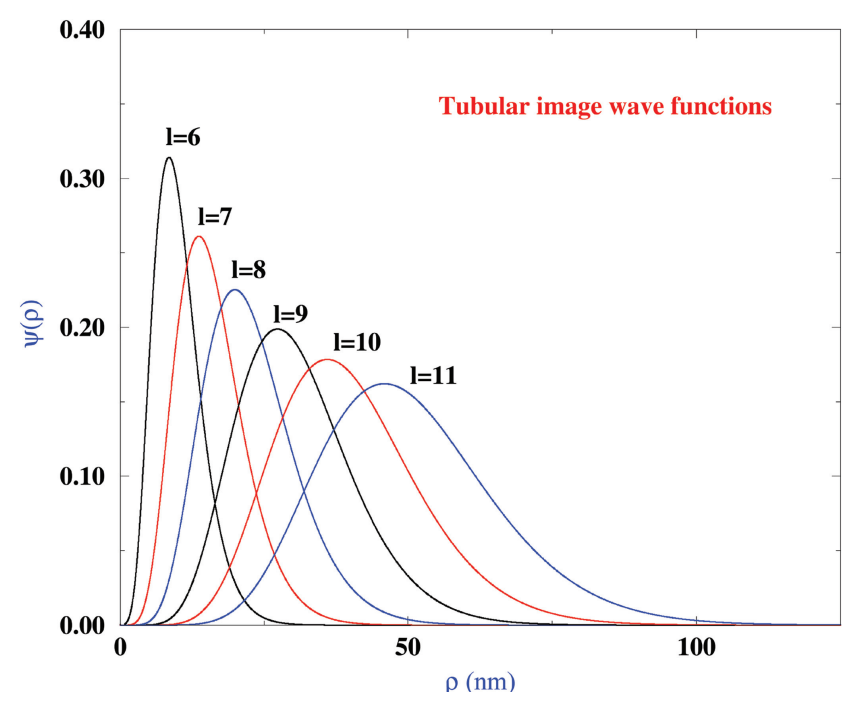

Fig. 8. The $n=1$ wave functions $\psi_{n=1, l}(\rho)$. From Ref. [32]. 


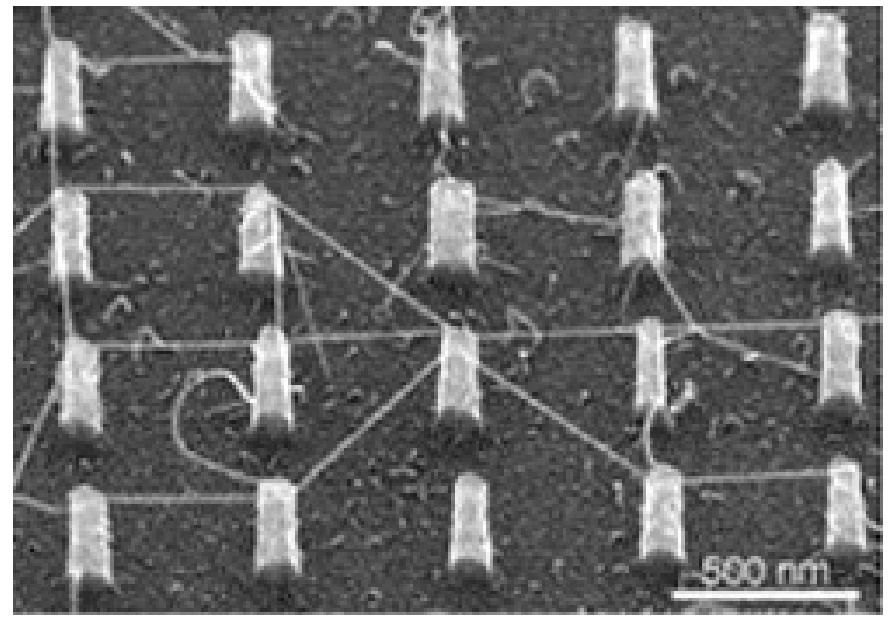

Fig. 9. Network of carbon nanotubes on 100-nm-scale silicon pillars. From Ref. [33].

approach, the cross section to capture electrons from Rydberg atoms scales geometrically as $n^{4}$. Both of these methods are ideal for populating the high angular momentum image states predicted here.

\section{Electronic Rydberg states near parallel arrays of nanotubes}

To observe Rydberg image states around conducting CNTs, nanotubes must be isolated from their surroundings. Such control of carbon nanotubes was demonstrated recently by a group at NTT who grew single-wall CNTs suspended from silicon pillars, etched by synchrotron radiation [33], see Fig. 9. The suspended CNTs were created in parallel arrays. This experiment raises also an interesting question: how would slow electrons propagate in such a lattice of conducting nanotubes?

Having derived the interaction of an electron with a single nanotube, the interaction of an electron with multiple, parallel nanotubes can be investigated. The simplest case is to consider an electron nearing two parallel nanotubes of radius $a$ whose axes are separated at a fixed distance. The total interaction potential $V_{T}(x, y)$ felt by the electron is simply,

$V_{T}=V\left(\rho_{1}\right)+V\left(\rho_{2}\right)$,

where $\rho_{1}$ and $\rho_{2}$ are the distances from the electron to the center of the two tubes, and $V\left(\rho_{1}\right)$ and $V\left(\rho_{2}\right)$ are the electron-nanotube interaction potentials. Because the $2 \mathrm{D}$ Schroedinger equation does not separate in any coordinate system, we chose to work in bipolar coordinates in which the boundary conditions are easy to apply. By employing a 2D basis set of B-splines, we have obtained energy eigenvalues and wavefunctions as a function of intertube separation and find striking similarities to Born-Oppenheimer potential energy curves for molecules [34]. For instance, we find gerade and ungerade states that exhibit energy splitting as a function of intertube distance. Further work will extend these ideas to the propagation of slow electrons through periodic arrays of CNTs and the formation of band structure in these arrays.

Interaction of charged particles and light with nanoscopic systems, such as carbon nanotubes, are interesting and may lead to surprising results and potential applica- tions. We have demonstrated that it is possible to use CNTs as nanomotor whose source of power is converted from absorption of light energy and angular momentum. Exotic electronic states should exist around CNTs with robust and familiar atomic properties.

\section{Acknowledgments}

This work was supported by the US National Science Foundation through a grant to the Institute for Theoretical Atomic, Molecular and Optical Physics at the Harvard-Smithsonian Center for Astrophysics.

\section{References}

1. Folk, J. A. et al., Science 299, 679 (2003).

2. Goldhaber-Gordon, D. et al., Nature 391, 156 (1998).

3. Pelton, M. et al., Phys. Rev. Lett. 89, 233602 (2002).

4. Kouwenhoven, L. P. et al., Science 278, 1788 (1997).

5. Iijima, S., Nature 354, 56 (1991).

6. Yu, M.-F. et al., Phys. Rev. Lett. 84, 5552 (2000).

7. Dresselhaus, M. S., Dresselhaus, G. and Eklund, P. C., "Science of Fullerenes and Carbon Nanotubes" (Academic Press Inc., San Diego, 1996).

8. Saito, R., Dresselhaus, G. and Dresselhaus, M. S., "Physical Properties of Carbon Nanotubes," (Imperial College Press, London, 1998).

9. Mintmire, J. W., Dunlap, B. I. and White, C. T., Phys. Rev. Lett. 68, 631 (1992).

10. Odom, T. W. et al., Nature 391, 62 (1998).

11. Wilder, J. W. G. et al., Nature 391, 59 (1998).

12. Bachilo, S. M. et al., Nature 298, 2361 (2002).

13. Dresselhaus, M. S. and Eklund, P. C., Adv. Phys. 49, 705 (2000).

14. Saito, R. (private communication).

15. Kral, P. and Sadeghpour, H. R., Phys. Rev. B 65, R-161401 (2002).

16. Goldstein, H., "Classical Mechanics," (2nd Ed., Addison-Wesley Publishing Co., Inc. Reading, 1980).

17. Benedict, L. X., Louie, S. G. and Cohen, M. L., Phys. Rev. B 52, 8541 (1995).

18. Ashkin, A., Phys. Rev. Lett. 24, 156 (1970); Renn, M. J., Pastel, R. and Lewandowski, H. J., Phys. Rev. Lett. 82, 1574 (1999).

19. Gallagher, T., "Rydberg Atoms," (Cambridge University Press, New York, 1994).

20. Clark, T. A., Naylor, D. A. and Davis, G. R., Astron. Astrophys. 361, L60 (2000).

21. Dalgarno, A., in "Rydberg States of Atoms and Molecules," (Eds. R. F. Stebbings and F. Barry Dunning) (Cambridge University Press, 1982).

22. "Recombination of atomic ions," (Ed. W. Graham, W. Fritsch, Y. Hahn and J. A. Tannis), NATO, ASI, Ser. B, vol. 296 (Plenum, NY 1992).

23. Gabrielse, G. et al., Phys. Rev. Lett. 89, 213401-1 (2002); Amoretti, M. et al., Nature 419, 456 (2002).

24. Killian, T. C. et al., Phys. Rev. Lett. 83, 4776 (1999); Killian, T. C. et al., Phys. Rev. Lett. 86, 3759 (2001).

25. Greene, C. H., Dickinson, A. S. and Sadeghpour, H. R., Phys. Rev. Lett. 85, 2458 (2000); Hamilton, E. L., Greene, C. H. and Sadeghpour, H. R., J. Phys. B: 35, L199 (2002).

26. Boyle, M. et al., Phys. Rev. Lett. 87, 273401 (2001).

27. Höfer, U. et al., Science 277, 1480 (1997).

28. Ortega, J. E. et al., Phys. Rev. B 49, 13859 (1994); Hill, I. G. and McLean, A. B., Phys. Rev. Lett. 82, 2155 (1999).

29. Kasperovich, V., Wong, K., Tikhonov, G. and Kresin, V. V., Phys. Rev. Lett. 85, 2729 (2000).

30. Platzman, P. M. and Dykman, M. I., Science 284, 1967 (1999); Glasson, P. et al., Phys. Rev. Lett. 87, 176802 (2001).

31. Lang, N. D. and Kohn, W., Phys. Rev. B 7, 3541 (1973); Shakeshaft, R. and Spruch, L., Phys. Rev. A 31, 1535 (1985).

32. Granger, B. E. et al., Phys. Rev. Lett. 89, 135506-1 (2002).

33. Homma, Y., Kobayashi, Y. and Ogino, T., Appl. Phys. Lett. 81, 2261 (2002).

34. Granger, B. E., Sadeghpour, H. R. and Kral, P. (to be published). 